\title{
Adesão medicamentosa em idosos em seguimento ambulatorial
}

\author{
M edicine adeshion in eldery people in an ambulatorial attendance
}

Fernanda A parecida Cintra ${ }^{1}$

M aria Elena Guariento ${ }^{2}$

Lilian Akemi M iyasaki ${ }^{1}$

${ }^{1}$ Departamento de Enfermagem, Faculdade de Ciências M édicas, Universidade Estadual de Campinas. RuaAlexander Fleming 40, distrito de Barão Geraldo. 13083-970 Campinas SP.

fernanda@fcm.unicamp.br ${ }^{2}$ Departamento deClínica M édica, Faculdadede Ciências M édicas, Universidade Estadual de Campinas.
Abstract The objectives of this study were to evaluate the elderly drug adhesion in an outpatient geriatric service linked to the State University Hospital of Campinas (São Paulo, Brazil) as well as to identify the related events to this adhesion. One hundred sixty five elders were submitted to an interview. It was used an instrument to collect information about the patients' identification, besides the self-reported state of health and drug utilization. The data were evaluated through the descriptive and comparative analysis. They showed that most of the elderly ( $88.5 \%$ ) have confirmed the drug adhesion and among them $91.1 \%$ were living with company. The elderly who were living alone presented three-time more chance of non-adhesion to the drug therapy ( $O R$ : 3.655). Those that have referred drug side effects presented seven-time more chance of nonadhesion (OR: 7.092). The associated events which better predict the drug non-adhesion were: "living alone" (OR: 4.346) and "side effects" (O R: 8.219).

Key words Drug use, Health of the elderly, Elderly
Resumo Este estudo objetivou avaliar a adesão ao tratamento medicamentoso em idosos em seguimento ambulatorial e identificar os fatores relacionados a esta adesão. Foram entrevistados 165 idosos em seguimento ambulatorial no H ospital das Clínicas da U niversidade Estadual de Campinas (Unicamp), São Paulo. U tilizou-se instrumento próprio, com informações relativas à identificação dos sujeitos, dados de saúde autorreferidos e relativos à terapêutica medicamentosa. Os dados foram submetidos às análises descritiva e de comparação. A maioria dos idosos revelou adesão ao tratamento $(88,5 \%)$, dentre os quais $91,1 \%$ moravam acompanhados. Os idosos que moravam sozinhos apresentaram três vezes mais chances de não aderência ao tratamento (OR: 3,655). Os efeitos colaterais dos medicamentos mostraram sete vezes mais chances de não aderência (OR: 7,092). Os fatores combinados que apresentaram maior chance de predizer a não adesão à terapêutica foram "mora sozinho" (OR: 4,346) e "efeitos colaterais" (OR: 8,219).

Palavras-chave U so de medicamentos, Saúde do Idoso, Idoso 
Introdução

A população idosa compreendeaproximadamente 17.800 .000 brasileiros, o que representa 9,5\% da população geral, estimada em 187 milhões de habitantes no último Censo de 2007, segundo o Instituto Brasileiro de Geografia e Estatística ${ }^{1}$. Este percentual revela o envel hecimento do país, decorrente do incremento da população idosa, e impõe desafios nas áreas da ciência, tecnologia e educação com vistas a possibilitar ao ser humano 0 alcance dos limites da longevidade de forma independente?

A velocidade com que o processo de transição demográfica vem ocorrendo no país remete a dificuldades para o Estado lidar com esse novo perfil epidemiológico. Nesse processo observase a substituição, entre as primeiras causas de morte, de doenças transmissíveis por doenças não transmissíveis e por causas externas, além do deslocamento da maior carga de morbimortalidade dos segmentos mais jovens para os mais envelhecidos. Modifica-se, assim, o perfil de morbimortalidade da população, com predomínio de doenças que exigem tratamento de longa duração, e que frequentemente são acompanhadas de complicações secundárias 3 .

A doença crônica, via deregra, requer mudanças no estilo de vida, especial mente entre os idosos, além do acompanhamento da evolução do quadro clínico que, se não controlado adequadamente, tende a agravar o prognóstico. 0 aumento da prevalência das doenças crônicas não transmissíveis, na idade avançada, coloca os idosos no grupo etário mais medicalizado da sociedade 4 .

O número elevado de portadores de doenças crônicas que utilizam os serviços de saúde repetidas vezes pode revelar, entre outros fatores, 0 seguimento inadequado da terapêutica medicamentosa. Na população idosa, esta inadequação da terapêutica, além deaumentar a demanda dos serviços de saúde, favorece a internação hospitalar ou contribui para ampliar a sua duraçãa ${ }^{5,6}$.

Apesar dos avanços tecnológicos para a redução da polifarmácia na maioria dos setores ligados às áreas de economia e de saúde, o custo gerado pela falta de adesão ao tratamento nos idosos mostra-se ainda elevado ${ }^{2}$. A esse respeito, grande número das hospitalizações que poderiam ser evitadas tem aumentado simultaneamente ao tempo de hospitalização, encarecendo ainda mais o tratamento.

$\mathrm{Na}$ literatura, é comum o uso concomitante dos termos "adesão" e "aderência" para expres- sar o cumprimento das ações terapêuticas. Apesar de interligados, os vocábulos "adesão" e "aderência" que se relacionam à mesma ação, ou seja, a de aderir, diferenciam-se por indicarem, respectivamente, 0 ato (adesão) e o efeito (aderência) dessa açãa?

Os problemas decorrentes da não adesão ao tratamento medicamentoso aumentaram nasúltimas décadas e têm preocupado os profissionais de saúde, dada a partici pação de fatores que influenciam o uso racional dos recursos terapêuti$\cos ^{8}$. Há quase duas décadas, já se chamava a atenção para a complexidade do uso inapropriado dos medicamentos entre os idosos, e destacava-sea importância de os profissionais de saúde orientarem os pacientes sobre o uso correto das drogas 9 .

Os estudos que abordam essa problemática, particularmente nos idosos, indicam vários fatores relacionados à não adesão ao tratamento, dentre os quais destacam-se: consumo elevado e uso prolongado, efeitos colaterais, desaparecimento dos sintomas, desconhecimento sobre os medicamentos, alto custo das medicações, falta de motivação, analfabetismo e distúrbios de memória ${ }^{5,9-13}$.

A partir dos dados obtidos pela Pesquisa Nacional por Amostra de Domicílio, em 1998, junto à população idosa brasileira, verificou-se que metade desta tinha um gasto médio mensal com medicamentos equivalentea $23 \%$ da sua ren$\mathrm{da}^{14}$. Este percentual, por si só, poderia justificar grande parcela das dificuldades enfrentadas por indivíduos nessa fai xa etária para o cumprimento da terapêutica medicamentosa.

Esse panorama levou ao desenvolvimento do presente estudo, que busca responder às seguintes indagações: os idosos em seguimento ambulatorial cumprem a terapêutica medicamentosa? Que fatores dificultam ou incentivam o cumprimento da terapêutica medicamentosa pelos idosos?

Espera-se, assim, obter elementos que facilitem a compreensão dessa problemática junto à população idosa, e fundamentem programas de intervenção e ações para a participação efetiva dos idosos na adesão ao tratamento.

Dessa forma, os objetivos do presente estudo são os seguintes:

- avaliar a adesão ao tratamento medicamentoso em idosos em seguimento ambulatorial;

- identificar os fatores relacionados à adesão ao tratamento medicamentoso em idosos em seguimento ambulatorial. 


\section{Método}

Realizou-se uma pesquisa do tipo descritiva/exploratória que permite obter informações acerca das características de sujeitos, grupos e instituições, ou ainda obter dados sobre a frequência de um determinado fenômeno ${ }^{15}$. Ela possibilitou verificar se os idosos cumprem a terapêutica medicamentosa e identificar os fatores relacionados a esse cumprimento.

O estudo foi desenvolvido no Hospital das Clínicas da Universidade Estadual de Campinas (Unicamp), São Paulo, nos ambulatórios de Cardiologia Geral, M edicina Interna e Oftalmologia. Estas especialidades foram eleitas devido ao número elevado de idosos em seguimento terapêutico.

Participaram do estudo 165 idosos com idade igual ou superior a 60 anos, de ambos os sexos. Esta faixa etária atende ao Estatuto do Idoso ${ }^{16}$, o qual assegura no artigo 15, capítulo IV, atenção integral à saúde do idoso, por meio de ações de preven ção, promoção eproteção da saúde, além de atenção às doenças que afetam preferencialmente essa classe populacional.

Foram considerados critérios de inclusão: estar em seguimento ambulatorial em uma ou mais especialidades aqui relacionadas; fazer uso contínuo de, pelo menos, um medicamento, por via oral ou tópica, pelo período mínimo de 15 dias4; demonstrar orientação auto e alopsíquica; apresentar capacidade para compreender e responder o instrumento de coleta de dados; e concordar em participar do estudo por meio da assinatura do Termo de Consentimento Livree Esclarecido.

Foram excluídos do estudo os pacientes que apresentaram pelo menos uma das seguintescondições: ter sido submetido a procedimento cirúrgico nos últimos 15 dias anteriores à coleta de dados; utilizar medicamentos pelo período inferior a 15 dias da participação na pesquisa; fazer uso de compostos homeopáticos, fitoterápicos ou chás4.

A exclusão de pacientes no período pós-ope ratório relacionou-se a uma possível motivação destes para o uso regular dosmedicamentos prescritos visando à recuperação do procedimento cirúrgico, considerado aqui um evento distinto da cronicidade das enfermidades manifestas na velhice.

0 tamanho da amostra ( $n=165)$ foi estabelecido conforme a indicação para análise multivariada ${ }^{17}$, que determina 15 sujeitos por variável preditora.

Os dados foram coletados por meio de um questionário elaborado a partir do Instrumento de Avaliação da Adesão ao Tratamento da Hipertensão ${ }^{18}$, no período dejaneiro a maio de 2006.

0 questionário foi dividido em: Partel - Identificação: contém dados de identificação dosidosos (nome, sexo, idade, estado civil e registro no hospital) e dados sociodemográficos (escolaridade, com quem mora, participação de cuidador, renda familiar mensal); Partell - Dados de saúde autorreferidos: aborda informações sobre o diagnóstico médico e condições clínicas associadas; Parte III - Dados relativos à terapêutica medicamentosa: relaciona os medicamentos em uso, forma de administração, adesão ao tratamento, efatores quedificultam ou incentivam os sujeitos ao tratamento.

Para o registro da adesão ao tratamento foi utilizada a classificação de M oreira e Araújo ${ }^{18}$ :

( ) Adesão extrema (AE): Afirma ter cuidado extremo com o horário e a forma de tomar os medicamentos;

( ) Limítrofe à adesão total (LA): Refere que, às vezes, se esquece de tomar os medicamentos, mas é raro;

( ) Intervalo médio de adesão (IM A): Refere claramente que tem dificuldade em tomar os medicamentos corretamente, devido aos efeitos colaterais;

( ) Limítrofeà não adesão total (LNA): Refere claramentequenão toma os medicamentos, apenas quando apresenta sintomas;

( ) Não adesão extrema (N AE): Refereclaramenteque não toma os medicamentos emenciona isto como sem importância.

As entrevistas foram realizadas individualmente por uma das autoras, em local reservado, durante a permanência dos pacientes nos ambulatórios eleitos para este estudo, nos períodos que antecederam as consultas médicas ou outros procedimentos.

A análise estatística foi realizada com o apoio do Serviço de Estatística da Comissão de Pesquisa da Faculdade de Ciências M édicas daUnicamp. Os dados foram inseridos no programa SAS System for Windows, versão 9.1.3, para as seguintes análises:

Descritiva: a partir da confecção de tabelas de frequências para as variáveis categóricas, e medidas de posição (média, mediana, mínima e máxima) e de dispersão (desvio-padrão) para as variáveis contínuas.

De comparação: para comparar as variáveis categóricas, utilizou-seo testeQ ui-quadrado $\left(\chi^{2}\right)$ ou, quando necessário, o teste Exato de Fisher. Para comparar as medidas contínuas ou ordenáveis entre dois grupos, foi empregado o teste 
de $M$ ann-W hitney. A identificação entre os fatores associados à não adesão medicamentosa foi realizada por meio da análise de regressão logística univariada e múltipla. Foi calculada a razão dechances (OddsRatio, OR) eo intervalo deconfiança (IC: 95\%). O nível de significância adotado para os testes estatísticos foi $5 \%$ ( $p$-valor: 0,05 ).

Foram garantidos aos sujeitos o sigilo eo anonimato da identidade, do registro no hospital e das informações obtidas por meio da entrevista.

\section{Resultados}

$\mathrm{Na}$ amostra estudada, a idade variou entre $60 \mathrm{e}$ 93 anos, com média de $71,9( \pm 7,8)$ anos epredomínio do sexo feminino $(66,0 \%)$. A maioria dos entrevistados era casada (58,8\%). 0 tempo mé dio de escolaridade foi de 3,0 ( $\pm 3,0$; mediana: $3,0)$ anos de estudo. Grande parte dos idosos morava acompanhada $(89,1 \%)$ e uma parcela significativa $(55,2 \%)$ referiu não ter cuidador. A renda familiar mensal informada mostrou mé dia de 2,4 ( $\pm 2,4$; mediana: 2,0) salários mínimos (SM ), oscilando entre um e vinte SM (Tabela 1).

Os idosos entrevistados estavam em seguimento clínico nos ambulatórios das seguintes especialidades médicas: M edicina Interna (41,8\%), Oftalmologia (33,9\%) e Cardiologia (24,2\%).

Com respeito aos diagnósticos médicos relatados pelos idosos, conforme as especialidades médicas, na M edicina Interna predominaram os distúrbios gastrintestinais, informados por $10,3 \%$ dos sujeitos, seguidos por diabetes mellitus relatado por $9,8 \%$. No ambulatório de $\mathrm{O}$ ftalmologia, a afecção mais referida foi a catarata, mencionada por $24,2 \%$. Na Cardiologia, houve um equilíbrio entre três dos diagnósticos autorreferidos: pós-operatório tardio de cirurgia cardíaca $(6,7 \%)$, hipertensão arterial earritmia cardíaca, com a mesma proporção $(6,1 \%)$.

Todos os idosos relataram, além da afecção correspondente ao diagnóstico médico da especialidadena qual estavam em seguimento ambulatorial, pelo menos uma comorbidade. A maior proporção $(58,2 \%)$ referiu apresentar acima de quatro condições clínicas associadas. 0 número decomorbidades/pacientereferido foi de 13 (média: 5,$3 ; \pm 2,4$ ).

Dentreas condições clínicas associadas, houve prevalência do déficit visual $(87,9 \%)$ e da hipertensão arterial sistêmica (80\%). As cardiopatias apareceram em $51,5 \%$ dos idosos, ea dislipidemia em $42,4 \%$. O utras afecções como diabetes mellitus e artrose foram observadas, na mesma proporção, em $40 \%$ dos entrevistados. 0 déficit auditivo e as vasculopatias foram relatados por $35,1 \%$ e $33,9 \%$, respectivamente.

O total de medicamentos em uso contínuo pelos entrevistados variou entre um e 12 , com média de 4,5 ( $\pm 2,4$; mediana: 4,0) medicamentos por idoso. Grande parte dos entrevistados (70,3\%) referiu utilizá-los sem auxílio ou supervisão. De forma equilibrada, $16,4 \%$ e $13,3 \%$ dos

Tabela 1. Descrição das variáveis sociodemográficas dos 165 idosos estudados. Campinas (SP), 2006.

\begin{tabular}{|c|c|c|c|c|c|c|}
\hline Variáveis & M édia ( $\pm D P$ ) & Mediana & $\begin{array}{l}\text { Variação } \\
\text { observada }\end{array}$ & $\begin{array}{c}\text { Distribuição em } \\
\text { categorias }\end{array}$ & $\mathrm{N}$ & $\%$ \\
\hline Idade & $71,9( \pm 7,8)$ & 72 & $60-93$ & - & - & - \\
\hline \multirow[t]{2}{*}{ Sexo } & - & - & - & $\mathrm{F}$ & 109 & 66,0 \\
\hline & - & - & - & M & 56 & 34,0 \\
\hline \multirow[t]{4}{*}{ Estado civil } & - & - & - & Casado & 97 & 58,8 \\
\hline & - & - & - & Viúvo & 49 & 29,7 \\
\hline & - & - & - & União consensual & 13 & 7,9 \\
\hline & - & - & - & Solteiro & 6 & 3,6 \\
\hline Escolaridade (anos de estudo) & $3,0( \pm 3,0)$ & 3 & $0-15$ & - & - & - \\
\hline \multirow[t]{2}{*}{ Arranjo familiar } & - & - & - & Mora acompanhado* & 147 & 89,1 \\
\hline & - & - & - & Mora sozinho & 18 & 10,9 \\
\hline \multirow[t]{2}{*}{ Cuidador } & - & - & - & Sim & 74 & 44,8 \\
\hline & - & - & - & Não & 91 & 55,2 \\
\hline $\begin{array}{l}\text { Renda familiar mensal } \\
(\text { em SM })^{* *}\end{array}$ & $2,4( \pm 2,4)$ & 2 & $1-20$ & - & - & - \\
\hline
\end{tabular}

*Com familiar ou não familiar; * SM = Salário mínimo. Valor vigente: $\mathrm{R} \$ 350,00$. 
idosos informaram que o uso de medicamentos era feito sob supervisão ou administrado por outra pessoa, respectivamente.

Ao serem interrogados sobre as dificuldades para a aquisição dos medicamentos, a referência à falta deles nas Unidades Básicas de Saúde apareceu em $63,6 \%$ das respostas, seguida dos gastos com medicamentos (62,4\%). Não relataram dificuldades 22,4\% dos entrevistados (Tabela 2).

Grande parte da amostra revelou adesão ao tratamento, a qual variou entre "adesão extrema” (53,9\%) e“limítrofeà adesão total” (34,6\%). Em menores proporções aparecem os idosos que relataram não adesão ao tratamento, com variação entre as classes "limítrofe à não adesão total" e "não adesão extrema" (Tabela 3).

Considerando o baixo número de idosos nas classes relacionadas à não adesão ao tratamento, foram criados os seguintes grupos: "adesão ao tratamento" (inclui "adesão extrema" e"limítrofe à adesão total") e "não adesão ao tratamento" (agrupamento de "limítrofe à não adesão total"; "intervalo médio de adesão" e "não adesão extrema").

Nos agrupamentos "adesão" e "não adesão", a maioria dos idosos concentrou-se na "adesão ao tratamento" (88,5\%), enquanto $11,5 \%$ deles permaneceram no grupo "não adesão ao tratamento".

Nos dados sociodemográficos identificados neste estudo, somente a variável "arranjo familiar" mostrou associação estatisticamente significativa com a adesão ao tratamento ( $p: 0,0382)$. Dentre os que aderiam ao tratamento, $91,1 \%$ (133/146) moravam acompanhados (com familiar ou não familiar) e 52,7\% (77/146) não ti-

Tabela 2. Frequência absoluta e relativa dos 165 idosos, segundo as dificuldades para a aquisição dos medicamentos. Campinas (SP), 2006.

\begin{tabular}{lrr}
\hline & N & $\%$ \\
\hline Falta dos medicamentos na UBS & 105 & 63,6 \\
Custo dos medicamentos & 103 & 62,4 \\
Número elevado de medicamentos & 38 & 23,0 \\
Efeitos colaterais & 12 & 7,3 \\
Limitação física para a compra dos & 2 & 1,2 \\
medicamentos & & \\
Uso prolongado de medicamentos & 1 & 0,6 \\
Outras dificuldades & 5 & 3,0 \\
Não apresenta dificuldade & 37 & 22,4 \\
\hline
\end{tabular}

*UBS: Unidade Básica deSaúde. nham cuidador. A variável "cuidador" mostrou apenas uma tendência de significância estatística (p: 0,0842).

Entre as dificuldades relatadas pelos idosos para a aquisição dos medicamentos, a variável "efeitos colaterais" foi a única que apresentou associação estatisticamente significativa com a adesão ao tratamento ( $p: 0,0056$ ). Dentre os idosos que não aderiam ao tratamento, 26,3\% (5/ 19) relatou efeitos colaterais dos medicamentos que utilizava.

$\mathrm{Na}$ análise de regressão logística univariada, houve associação positiva entre a não adesão ao tratamento e as variáveis "mora sozinho" e"efeitos colaterais". Os idosos que moravam sozinhos apresentaram três vezes mais chances de não aderência ao tratamento (O R: 3,655; IC: 1,135 a $11,766)$. Da mesma forma, aqueles que relataram os efeitos colaterais dos medicamentos mostraram sete vezes mais chance de não aderência ao tratamento (O R: 7,092; IC: 1,987 a 25,315). A variável "número de condições clínicas associadas" apresentou apenas uma tendência de significância estatística na associação com a não adesão ao tratamento (p: 0,0592) (Tabela 4).

Os fatores combinados que apresentaram mai or chance de predizer a não adesão ao tratamento foram "mora sozinho" (OR: 4,346; IC: 1,271 a 14,857) e "efeitos colaterais" (OR: 8,219; IC: 2,199 a 30,716) (Tabela 5).

Para ilustrar os dados relativos ao cumprimento da terapêutica medicamentosa, realizouse uma análise qualitativa dos depoimentos dos idosos com respeito aos fatores que dificultavam ou incentivavam esse cumprimento. Para tanto, foram agrupados os motivos com sentidos e significados comuns que melhor expressavam as dificuldades e motivação manifestas pelos idosos.

Tabela 3. Distribuição dos idosos conforme a adesão ao tratamento medicamentoso. Campinas (SP), 2006.

\begin{tabular}{lrr}
\hline & N & \multicolumn{1}{c}{$\%$} \\
\hline Adesão extrema & 89 & 53,9 \\
Limítrofe à adesão total & 57 & 34,6 \\
Limítrofe à não adesão total & 15 & 9,1 \\
Intervalo médio de adesão & 2 & 1,2 \\
Não adesão extrema & 2 & 1,2 \\
Total & 165 & 100,0 \\
\hline
\end{tabular}


Tabela 4. Resultados da análise de regressão logística univariada das variáveis associadas à não adesão ao tratamento. Campinas (SP), 2006.

\begin{tabular}{|c|c|c|c|}
\hline Variáveis & $O \mathbf{R}^{*}$ & $\begin{array}{l}\text { Não adesão ao tratamento } \\
\text { IC } 95 \%^{* *}\end{array}$ & $p$-valor ${ }^{* * *}$ \\
\hline Sexo & - & - & - \\
\hline Masculino $x$ Feminino & 0,482 & $0,152-1,528$ & 0,2151 \\
\hline Idade (anos) & 0,998 & $0,938-1,061$ & 0,9382 \\
\hline $70-79 \times 60-69$ & 1,650 & $0,600-4,537$ & 0,3318 \\
\hline$\geq 80 \times 60-69$ & 0,391 & $0,046-3,356$ & 0,3921 \\
\hline Escolaridade & 1,008 & $0,862-1,178$ & 0,9210 \\
\hline Estado civil & - & - & - \\
\hline Solteiro/viúvo x casado/união consensual & 0,914 & $0,327-2,550$ & 0,8631 \\
\hline Arranjo familiar & - & - & - \\
\hline Mora sozinho $\mathrm{x}$ acompanhado & 3,655 & $1,135-11,766$ & 0,0298 \\
\hline Cuidador & - & - & - \\
\hline Sim $x$ Não & 2,509 & $0,859-7,326$ & 0,0325 \\
\hline Renda familiar mensal & 0,861 & $0,619-1,196$ & 0,3712 \\
\hline $\mathrm{N}$ ㅇ de condições clínicas associadas & - & - & - \\
\hline Sim x Não & 1,200 & $0,993-1,450$ & 0,0592 \\
\hline Gastos com medicamentos & - & - & - \\
\hline Sim x Não & 2,472 & $0,781-7,819$ & 0,1236 \\
\hline $\mathrm{N} \cong$ elevado de medicamentos & - & - & - \\
\hline Sim x Não & 1,223 & $0,410-3,646$ & 0,7180 \\
\hline Efeitos colaterais & - & - & - \\
\hline Sim x Não & 7,092 & $1,987-25,315$ & 0,0025 \\
\hline Falta dos medicamentos na UBS ${ }^{* * * *}$ & - & - & - \\
\hline Sim x Não & 2,333 & $0,737-7,386$ & 0,1495 \\
\hline Não apresenta dificuldade & - & - & - \\
\hline Sim $x$ Não & 5,890 & $0,759-45,686$ & 0,0898 \\
\hline $\mathrm{N} \cong$ total de medicamentos em uso & - & - & - \\
\hline Sim x Não & 1,101 & $0,915-1,324$ & 0,3103 \\
\hline
\end{tabular}

Tabela 5. Resultado da regressão logística múltipla das variáveis associadas à não adesão ao tratamento. Campinas (SP), 2006.

\begin{tabular}{lrrr}
\hline \multicolumn{1}{c}{ Variáveis } & OR $^{*}$ & $\begin{array}{r}\text { Não adesão ao tratamento } \\
\text { IC 95\%** }\end{array}$ & p-valor $^{* * *}$ \\
\hline Intercepto & - & - & $<0,0001$ \\
Arranjo familiar & - & - & - \\
$\quad$ M ora sozinho x Acompanhado & 4,346 & $1,271-14,857$ & 0,0191 \\
Efeitos colaterais & - & $2,199-30,716$ & - \\
$\quad$ Sim x Não & 8,219 & 0,0017 \\
\hline
\end{tabular}

*OR: razão de chances (odds ratio); *IC: intervalo de confiança em nível de 95\%; *** p-valor: 0,0001.

Com respeito aos motivos que dificultavam o cumprimento do tratamento, os idosos apontaram os efeitos colaterais dentre os quais destacaram-se: "dor no estômago" e "constipação in- testinal". A associação entre os efeitos colateraise a ausência de companhia de outra pessoa para morar podeser verificada no depoimento deuma das idosas: Como moro sozinha, não tomo (os 
medicamentos) por causa dos efeitos ruins. A falta de companhia para morar também apareceu associada à perda da memória: M oro sozinha, não lembro de tomar (os medicamentos).

Outros fatores também chamaram a atenção, como a dificuldade financeira para a compra dos medicamentos, associada ao custo elevado, bem como o número elevado de medicamentos: Sinto muito sono, são muitos remédios para lembrar.

Os idosos também apontaram facilitadores da adesão ao tratamento. Dentre eles, destaca-se o apoio da família como importante coadjuvante: $M$ eu marido não deixa esquecer dos remédios... Ele sabe direitinho os horários. A necessidade do cumprimento da terapêutica, bem como o medo de agravo à saúde e a vontade de viver, aparece ram de forma explícita nos depoimentos: ...não pode ficar sem tomar, porque sem tomar piora; ...vontade de viver, de não ficar doente. Faço caminhada todas as manhãs para a osteoporose, para melhorar a saúde.

\section{Discussão}

A análise do presente estudo mostra que a maioria dos idosos entrevistados apresentava adesão ao regime terapêutico, sendo que cerca de metade deles afirmou ter cuidado extremo com o horário e a forma de tomar os medicamentos. Esta adesão é coerente com outras investigações na população idosa $a^{9,11,13,19} \mathrm{e}$ em adultos não necessariamente idosos ${ }^{12,18}$. No entanto, num estudo que avalia o grau de cumprimento da prescrição médica em idosos, considerando os motivos e características biossociais, somente $8,4 \%$ da amostra cumpriam totalmente 0 tratamento ${ }^{5}$.

A literatura acerca do uso de medicamentos na população idosa aponta diversos fatores que influenciam a não adesão ao tratamento medicamentoso. Dentreeles, destacam-se os efeitos colaterais, a falta de informações sobre a terapêutica, 0 alto custo e o número elevado de medicamentos, além das características socioeconômicas $5^{5,9,11,13,19-22}$.

$\mathrm{Na}$ presente investigação, não foi encontrada associação entre o uso incorreto das medicações e as variáveis: idade, sexo, estado civil, escolaridade e renda mensal. Entretanto, observou-se forte associação entre a não adesão e 0 arranjo familiar "mora sozinho". A participação da família ou do cuidador mostra-se importante para o cumprimento da terapêutica pelos idosos, uma vez que com o avançar da idade eles tendem a se tornar mais dependentes devido aos déficits cog- nitivo e fisiológico, característicos dessa fase da vida $5,9,11,13$.

Estudos que analisam a situação do idoso no Brasil indicam a presença de pelo menos uma condição clínica crônica em pessoas com idade igual ou superior a 60 anos $^{2,13,14}$. De forma semeIhante, todos os idosos deste estudo revelaram a presença de pelo menos uma condição clínica associada, além do diagnóstico principal. A maior prevalência foi de déficit visual, seguida da hipertensão arterial sistêmica. A alta proporção da hipertensão arterial sistêmica já era esperada, tendo em vista que esta afecção crônica é comum nas faixas etárias mais elevadas $2-5,13,14,19-21$. Contudo, chama a atenção o número expressivo de idosos que relataram apresentar queda na acuidade visual, considerando as implicações da baixa visão no cumprimento da terapêutica medicamentosa, o que sugere futuras investigações a esse respeito.

Todos os idosos entrevistados informaram utilizar pelo menos um medicamento, com média de 4,5 medicamentos por sujeito. Este dado aproxima-se aos resultados encontrados na literatura 3,4,13,19,21,22. A quantidade diária de medicamentos a ser consumida pode originar erros na sua administração, particularmenteentre os idosos. 0 uso de vários comprimidos ao dia em horários distintos pode ser dificultado pelo esquecimento, trabalho e déficit cognitivo ${ }^{5,20}$. No presente estudo, a maioria dos entrevistados referiu tomar os medicamentos "por conta própria", sem qualquer forma de auxílio ou supervisão. Isto remete à necessidade de orientação profissional de forma diferenciada aos idosos, especialmente na prescrição das medicações ${ }^{13,23}$.

A dificuldade para adquirir os medicamentos foi relatada por grande parcela dos entrevistados. A falta de medicamentos nas Unidades Básicas de Saúde foi o principal motivo identificado para esta dificuldade. Isto revela uma possível dependência desses sujeitos em relação à Rede Básica de Saúde, justificada, em parte, pela baixa renda familiar mensal informada (média de 2,4 salários mínimos). Este achado está em consonância com o quefoi encontrado por Lima-Costa et al. ${ }^{14}$ na avaliação de idosos residentes no município de Bambuí (MG).

No Brasil, as condições financeiras precárias da população idosa, associadas aos gastos com medicamentos, vêm sendo destacadas na literatura, tendo em vista as implicações na adesão terapêutica ${ }^{11,13,21,23}$. O uso em doses inferiores ao que recomenda a prescrição médica por iniciativa própria, por partedosidosos, érevelado como tentativa de "economizar" a medicação, especial- 
mente no final dos meses. Esta conduta é motivada pelo medo da falta da medicação nas Unidades Básicas de Saúde, bem como pelas condições financeiras precárias para adquiri-la ${ }^{13}$. O utras estratégias são utilizadas pelos idosos para a baixa renda: uso decrédito, substituição da compra de alimento pelo medicamento, ou até mesmo 0 não cumprimento do tratamento ${ }^{5,11,20,21}$. Também são relatados estudos europeus acerca da não adesão aos medicamentos. $\mathrm{N}$ a Alemanha e na República Tcheca, por exemplo, os serviços públicos de saúde são eficientes, porém o alto custo das medicações e a menor renda da população idosa são apontados como fatores que influenciam a não aderência ${ }^{19}$.

Esta investigação mostrou forte associação entre os efeitos colaterais dos medicamentos e a não adesão à terapêutica. Estudos revelam o medo de reações adversas causadas pelas medicações como um dos fatores para o não cumprimento da prescrição médica5,11,13. Esse não cumprimento pode, em alguns casos, estar associado à manifestação de reações indesejáveis, nem sempre compreendidas pelo paciente. Entretanto, chamam a atenção as associações inadequadas e comumente realizadas pelos idosos que acabam por responsabilizar a medicação pelos efeitos indevidamente atribuídos a ela, suspendendo-a desnecessariamente ${ }^{20}$.

A análise do presente estudo revela que os idosos que moram sozinhos apresentam três vezes mais chance de não aderência ao tratamento.
De forma semelhante, aqueles que relataram os efeitos colaterais dos medicamentos mostraram sete vezes mais chance de não aderir ao tratamento. Além disso, os fatores "morar sozinho" e "efeitos colaterais", quando combinados, são os que melhor predizem a não adesão ao tratamento. Estes achados corroboram outras investigações sobre 0 tema $a^{5,9,11,13,19-21}$ e reiteram a demanda de atenção para essa problemática no cuidado à população idosa.

\section{Conclusão}

Apesar das limitações apresentadas pelos idosos, a grandemaioria revelou cumprir a terapêutica medicamentosa, possivelmente motivada pelo "medo de agravo à saúde" e pela "vontade de viver".

A condição de morar sem companhia e os efeitos colaterais dos medicamentos, fortes preditores para a não adesão à terapêutica, sinalizam a relevância do apoio de outras pessoas (familiares ou não), que podeser materializado pela convivência com osidosos. Isso remeteà necessidade de atenção individualizada a essa população, com vistas a identificar possíveis fatores para a não adesão ao tratamento, bem como incrementar ações efetivas para facilitar esta adesão.

A educação em saúde aos idosos e familiares, de forma individual ou em grupo, apresenta-se como uma estratégia que possibilita a participação e o envolvimento na terapêutica²3.

\section{Colaboradores}

FA Cintra eM E Guariento participaram igualmente de todas as etapas da elaboração do artigo; LA M iyasaki participou do projeto, aplicação do instrumento (entrevistas) e análise dos resultados. 


\section{Referências}

1. Instituto Brasileiro de Geografia e Estatística (IBGE) [site da Internet] [acessado 2008 mar 3]. Disponível em: http://www.ibge.gov.br/home/estatistica/ populacao/contagem2007/defaulttab.shtm

2. Veras R. Em busca de uma assistência adequada à saúde do idoso: revisão da literatura e aplicação de um instrumento de detecção precoce e de previsibilidade de agravos. Cad Saude Publica 2003; 19(3):705-715.

3. Chaimowicz F. A saúde dos idosos brasileiros às vésperas do século XXI: problemas, projeções e alternativas. Rev Saude Publica 1997; 31(8):184-200.

4. M osegui GBG, Rozenfeld S, Veras RP, Vianna CM M . Avaliação da qualidade do uso de medicamentos em idosos. Rev Saude Publica 1999; 33(5):437-444.

5. Teixeira JJV, Spínola AW P. Comportamento do paciente idoso frente à aderência medicamentosa. Arq Geriatr Gerontol 1998; 2(1):5-9.

6. Nóbrega OT, Karnikowski M GO. A terapia medicamentosa no idoso: cuidados na medicação. Cien Saude Colet 2005; 10(2):309-313.

7. Ferreira ABH. N ovo Dicionário da Língua Portugue sa. $2^{\mathrm{a}}$ ed. Rio de Janeiro: N ova Fronteira; 1986.

8. Leite SN, Vasconcellos M PC. Adesão à terapêutica medicamentosa: elementos para a discussão de conceitos e pressupostos adotados na literatura. Cien Saude Colet 2003; 8(3):775-782.

9. Bernstein LR, Folkman S, Lazarus RS. Characterization of the use and misuse of medications by an elderly, ambulatory population. Medical Care 1989; 27(6):654-663

10. Brawley LR, Culos-Reed SN. Studying adherence to therapeutic regimens. Control Clin Trials 2000; 21(5):156-163.

11. Firmo JAO, Lima-Costa M F, Uchôa E. Projeto Bambuí: maneiras de pensar e agir de idosos hipertenSOs. Cad Saude Publica 2004; 20(4):1029-1040.

12. Guedes NG, Costa FBC, Moreira RP, Chaves ES, Araújo TL. Crises hipertensivas em portadores de hipertensão arterial em tratamento ambulatorial. Rev Esc Enf USP 2005; 39(2):181-188.

13. Vasconcelos FF, Victor JF, M oreira TM M, Araújo TL. Utilização medicamentosa por idosos de uma Unidade Básica de Saúde da Família de FortalezaCE. Acta Paul Enferm 2005; 18(2):78-83.
14. Lima-Costa M F, Barreto SM, Giatti L. Condições de saúde, capacidade funcional, uso de serviços de saúde e gastos com medicamentos da população idosa brasileira: um estudo descritivo baseado na Pesquisa Nacional por Amostra de Domicílios. Cad Saude Pública 2003; 19(3):735-743.

15. Lobiondo-Wood G, Haber J. N ursing research: methods, critical appraisal and utilization. $3^{\text {rd. }}$ ed. St. Louis: Mosby; 1994.

16. Brasil. Ministério da Saúde. Estatuto do Idoso. 1a ed. Brasília: Ministério da Saúde; 2003.

17. Stevens J. Applied multivariate statistics for the social sciences. $2^{\text {nd }}$ ed. Hillsdale: Lawrence Erlbaum Associates; 1992.

18. Moreira TMM, Araújo TL. Verificação da eficácia de uma proposta de cuidado para aumento da adesão ao tratamento da hipertensão arterial. Acta Paul Enferm 2004; 17(3):268-277.

19. Cooper C, Carpenter I, Katona C, Schroll M, Wagner C, Fialova D, Livingstone G. The AdH OC Study of Older Adults' Adherence to Medication in 11 Countries. Am J Psychiatry 2005; 13(12):1067-1076.

20. Diaz RB. Adesão ao tratamento medicamentoso em pacientes idosos. In: Papaléo Netto M. Gerontologia. São Paulo: Atheneu; 1996. p. 230-241.

21. Loyola-Filho AI, Uchoa E, Firmo JOA, Lima-Costa $M A$. Estudo de base populacional sobre o consumo de medicamentos entre idosos: Projeto Bambuí. Cad Saude Publica 2005; 21(2):545-553.

22. Nir Z, Weisel-Eichler A. Improving knowledge and skills for use of medication by patients after stroke: evaluation of a nursing intervention. Am J Phys M ed Rehabil 2006; 85(7):582-592.

23. Cintra FA, Sawaia BB. A significação do glaucoma e a mediação dos significados de velhice na perspectiva vygotskiana: subsídios para a educação à saúde. Rev Esc Enf USP 2000; 34(4):339-346.

Artigo apresentado em 16/08/2007

Aprovado em 08/02/2008

Versão final apresentada em 08/03/2008 